\title{
RELACIÓN IGLESIA MUNDO EN LA EXPERIENCIA DE LAS PRIMERAS COMUNIDADES CRISTIANAS
}

Luzio Uriarte

Con frecuencia Iglesia y mundo aparecen como dos términos contrapuestos. La Iglesia como lugar de salvación y el mundo como ámbito de perdición. Este planteamiento, por lo demás caricaturesco, va siendo superado en el imaginario cristiano, aunque ciertamente todavía permanece un sustrato dualista. Sin embargo, los dos términos se necesitan entre sí y tienen su sentido dentro de la historia de salvación que acontece en medio de la humanidad. El Concilio Vaticano II ha supuesto el respaldo a una mirada más positiva del mundo y el impulso para buscar caminos de encuentro y diálogo.

En este escrito pretendemos iluminar esta temática, refiriéndonos a la experiencia que tuvieron las primeras comunidades cristianas en su encuentro con el mundo. Partiremos ubicando el significado de la relación Iglesia-mundo desde la perspectiva de la tarea evangelizadora de la Iglesia, para posteriormente adentrarnos en las diversas formas cómo las primeras comunidades cristianas se plantearon su relación con el mundo y las consecuencias que tuvieron estos planteamientos.

\section{A. Punto de partida \\ 1. Importancia de la relación Iglesia-mundo para comprender la Iglesia}

La relación que se establece entre la Iglesia y el mundo ${ }^{1}$ es una de las claves de comprensión de la esencia del acontecimiento eclesial; en realidad esta afirmación no es más que la consecuencia misma de la identidad original y fontal que tiene

Para identificar en qué sentido utilizamos el concepto mundo se puede consultar en G. BOF, "Mundo" en G. BARBAGLIO,-S. DIANICH, Nuevo Diccionario de Teologia, Madrid, Cristiandad $1982,1137-1152$.

Dentro de la Biblia el término "mundo" tiene una acepción negativa y otra neutra. En sentido negativo se utiliza como la realidad marcada por el pecado que se opone al plan salvador de Dios (1Cor 2,12$)$ y se concreta en la oposición Dios-mundo. En sentido neutro el término "mundo" expresa todo el orden de la creación ( $\mathrm{Gal} 4,3$; Flp 2, 15); también designa el ambiente en el que se desenvuelve la vida del hombre (Rom 4, 13; 1Cor 14, 10) asi como el conjunto de condiciones y posibilidades de vida ofrecidas al hombre en la tierra (1Cor 3,$22 ; 4,13)$. En un sentido más amplio se puede hablar de mundo como el entorno generado por la misma actividad humana en cuyo seno va creciendo el ser humano; así nos referimos al entorno social, cultural, económico y politico. La tradición juánica presenta un uso un tanto diverso a este sentido y que tendremos la oportunidad de analizar más adelante. 
la Iglesia; se trata de una identidad relacional, que nace y crece en el seno de los encuentros que son significativos para ella. La relación originante de su existencia, y que por cierto configura su misterio ${ }^{2}$, es la convocatoria que Dios Padre realiza en Jesús y que es permanentemente actualizada por la fuerza del Espíritu Santo; en esta convocatoria la Iglesia está llamada a servir a la humanidad y ser testigo fiel del proyecto de Dios en medio de los hombres y mujeres. En esencia ese proyecto consiste en formar parte y actuar familia de Dios, hijos de un mismo Padre y hermanos en el Hermano mayor. El acontecimiento trinitario originante de la existencia de la Iglesia implica necesariamente una relación de encuentro con los hombres entre los que está llamada a ser testimonio vivo y eficaz de la voluntad salvífica de Dios. Es decir, la identidad de la Iglesia le lleva necesariamente a relacionarse con el mundo, pues en él encuentra el motivo de su existencia y le es posible desarrollar su identidad en la medida que cumple su función evangelizadora; al margen del mundo la Iglesia no puede ser testigo y testimonio de la 'Buena Noticia' de Jesús y de su 'Reino de justicia'; como dice Pablo VI: "Nosotros queremos confirmar una vez más que la tarea de la evangelización de todos los hombres constituye la misión esencial de la Iglesia (...). Evangelizar constituye, en efecto, la dicha y vocación propia de la Iglesia, su identidad más profunda"3.

Sabemos que el mundo propio de la humanidad no es una realidad homogénea; hoy más que nunca somos conscientes de la gran diversidad cultural que existe a lo largo de las diferentes sociedades; como ha puesto de relieve la Antropología, la cultura no es una realidad exterior y prescindible en la persona; por el contrario, la cultura moldea las formas de pensar, sentir y actuar de los seres humanos; cada cultura lleva a las personas que participan en ella a tener una determinada y especffica comprensión del mundo. La Iglesia, para llegar al corazón del hombre y de la humanidad, necesariamente tiene que entrar en relación con las diferentes culturas en las que vive la persona; debe conectar con el corazón de la cultura. Sin la mediación cultural, el evangelio no sería significativo para un determinado pueblo; por ello, el Espíritu en Pentecostés hace que cada uno de los oyentes escuche y comprenda la predicación apostólica en su propia lengua, es decir dentro de su propia forma de percibir el mundo (cfr Hch 2, 5-11); la evangelización no hace que las personas salgan de su cultura sino que se adapta a cada sociedad; evidentemente esta adaptación no es mimética ni automática sino un lento proceso de discernimiento a través del cual se produce una acogida y una potenciación de los valores evangélicos que están presentes en esa cultura al mismo tiempo que se critican y criban los modos de pensar, sentir y actuar que van en contra de la plena realización del ser humano según el proyecto de Dios. Un elemento que complejiza aún más este discernimiento es que la Iglesia siempre está viva dentro de una determinada sociedad, y desde el primer momento participa de una cultura (inicialmente la cultura judia desde la que parten las primeras comunidades) a través de la

El término misterio lo utilizamos en el sentido paulino, equivalente al término latino de sacramento; es importante evitar una mala comprensión de este término, por lo demás muy utilizado en la literatura actual, aunque no siempre con el mismo sentido. Es decir, por misterio entendemos el plan salvifico de Dios para con la humanidad que se va desarrollando dentro de la historia de los hombres; en dicho plan la Iglesia tiene una misión especifica dentro de esta iniciativa salvifica de Dios. 
cual expresa la 'Buena Noticia'; al llegar a otra cultura, la Iglesia no lleva un 'evangelio sin cultura'; por el contrario está comprendido y expresado en las formas propias de un determinado molde; la relación con otra sociedad implica también el discernimiento de determinadas formas, hábitos, instituciones que ha ido adquiriendo la Iglesia.

\section{Experiencia de las primeras comunidades cristianas como referencia paradigmática.}

Esta relación Iglesia-mundo y la tensión que le acompaña está presente desde los primeros pasos de las comunidades cristianas. Sin embargo es evidente que la experiencia que realizaron estas comunidades cristianas es especialmente importante para el conjunto de la Iglesia y a mi entender revisten hoy un significado particular, dado el contexto que vivimos en el mundo contemporáneo.

La experiencia misionera que realizaron los primeros grupos cristianos constituye el primer movimiento de salida hacia un mundo que no compartía la misma fe. Este momento en la vida de la Iglesia es muy significativo y de gran trascendencia histórica; en primer lugar porque si esos primeros seguidores de Jesús, a la luz del encuentro con el Resucitado y guiados por el Espíritu Santo, no hubieran transcendido las fronteras de su propia cultura y mundo religioso inicial, hoy el cristianismo se reduciría al ámbito de influencia judio (en el caso que hubiera podido subsistir de forma diferenciada); con ello se pone de manifiesto una de las características esenciales que tiene toda comunidad de seguidores de Jesús: el anuncio de la 'Buena Noticia' en el mundo es esencial a su vida; cuando se pierde esta dimensión evangelizadora, se debilita la identidad comunitaria y corre el riesgo de desaparecer; a la luz de esta experiencia inicial, el anuncio no es una tarea más sino una dimensión en la vida de los creyentes; la realización de esta dimensión pone a la comunidad creyente inmediatamente en contacto con el mundo externo, con los no creyentes. Pero no sólo es relevante la constatación de la existencia de esta dimensión evangelizadora en las primeras comunidades cristianas; también resulta muy significativo el modo de la evangelización; es decir, la forma que tuvieron de valorar su entorno y de relacionarse con él así como las diversas estrategias que utilizaron para anunciar y extender la 'Buena Noticia'. En este punto se pone ya de manifiesto, aunque sea de forma inicial, una de las tensiones que acompaña siempre la realización de la dimensión evangelizadora; todas las comunidades presentes en el NT participan de una determinada cultura y de unas formas religiosas muchas de hechas influidas o incluso heredadas del judaísmo. Cuando salen al encuentro de otras sociedades y anuncian la 'Buena Noticia' lo hacen desde su marco cultural inicial; las formas como asumieron la diversidad cultural y religiosa, asi como las consecuencias que estas formas tuvieron para la comprensión y la organización de la comunidad tienen gran trascendencia para comprender el dinamismo eclesial.

En todo esto hay otro aspecto que hemos de tener muy en cuenta a la hora de valorar la importancia de estas primeras experiencias de inculturización de la fe. Los textos del Nuevo Testamento son normativos para todos los cristianos; en ellos, cada seguidor de Jesús y la Iglesia bebe permanentemente para alimentar la fe y buscar los caminos de su concreción histórica en el mundo que le toca vivir; pues bien, estos textos, esenciales para el cristiano de todos los tiempos y lugares, na- 
cen en el seno de las primeras comunidades cristianas las cuales precisamente se encuentran realizando esta experiencia de evangelización y apertura al mundo; es decir la experiencia que realizaron de evangelización y de encuentro con el mundo es el contexto en el que se escriben los textos del NT y se discierne el canon; por tanto, esta experiencia misionera está presente en la redacción misma de la Sagrada Escritura, al mismo tiempo que configura un contexto para su discernimiento.

Todo apunta a que tenemos que valorar muy atentamente el camino y la experiencia evangelizadora que realizaron las primeras iglesias cristianas puesto que se convierte en paradigma de toda comunidad de seguidores de Jesús.

Finalmente, entre las diversas condiciones socio-culturales que confluyen en el tiempo de las primeras comunidades cristianas hay una que resulta especialmente relevante para los cristianos contemporáneos. Hasta hace pocas décadas, muchas sociedades estaban marcadas por ser mayoritariamente cristianas (católicas, protestantes, ortodoxas...); había una cierta compenetración entre cultura, sociedad y religión cristiana, aunque no fuera más que por el hecho de que no ser cristiano estaba mal visto. Hoy, en cambio, cada vez está más claro que vamos hacia un mundo plural, donde todas las sociedades y culturas entran en mutua relación e influencia recíproca; al mismo tiempo, los grandes paradigmas interpretativos de la realidad han ido cayendo, o por lo menos, ya no logran un amplio consenso. Esto hace que la religión cristiana se perciba socialmente como una religión más entre otras, en donde los ciudadanos pueden elegir entre diversas posibilidades, muchas de ellas aceptadas con normalidad; se pone en evidencia la multiplicidad de culturas y de valores, incluso dentro de una misma sociedad; la situación puede resultar bastante chocante para la iglesias que han estado acostumbradas a vivir en sociedades mayoritariamente cristianas y en gran parte ayudadas por los poderes públi$\cos$; no es tan chocante para las iglesias que han vivido como comunidades minoritarias en sociedades caracterizadas por otras religiones y culturas de matriz no cristianas. Sin lugar a dudas estamos en un tiempo de cambio y de aprendizaje, tiempo de discernimiento y de purificación de la fe. Mirando la historia de la Iglesia no es una situación nueva; las comunidades del NT viven como grupos pequeños y minoritarios en sociedades muy plurales, abiertas a muchas influencias culturales, con marcos valóricos contradictorios y en medio de la presencia de diversas religiones. La forma como comprendió el mundo y realizó su misión esta primera Iglesia, puede resultar especialmente significativo e inspirador para la comunidades contemporáneas; es una referencia esencial para discernir caminos, estimular búsquedas, superar desazones...

\section{B. DiVERsas experiencias en las PRIMERAS Comunidades Cristianas.}

Cuando nos acercamos a las experiencias comunitarias que subyacen a los actuales textos del Nuevo Testamento descubrimos una pluralidad de situaciones y de estrategias que resultan muy estimulantes para nuestra reflexión. Ciertamente los textos sagrados no pretenden darnos una crónica histórica de acontecimientos de la época; tampoco tienen como interés hablar de las comunidades que están a la base de los escritos. La intención es claramente teológica; se busca comprender el significado de los acontecimientos a la luz de la fe y con ello orientar la praxis de las 
comunidades y de los creyentes del momento; son al mismo tiempo testimonios de fe que invitan a vivir la fe con toda profundidad y radicalidad; finalmente son un estímulo para vivir la fe con aguante y esperanza frente a las dificultades que se presentan en la coyuntura histórica del momento. Por tanto no vamos a encontrar una reflexión elaborada que nos responda inmediatamente a nuestros interrogantes; vale decir, no hay una reflexión estructurada y sistemática sobre el mundo sociocultural en que les toca vivir y la forma de cómo evangelizarlo; tampoco hay un estudio sistemático de cómo organizar la comunidad o de planteamientos y estrategias en la forma de estar y relacionarse con el mundo. Sin embargo, indirectamente, $y$ a partir de las reflexiones y narraciones que se nos presentan, se pueden inferir diversidad de contextos socio-culturales, distintas sensibilidades comunitarias a la hora de estar presente en el mundo y una rica pluralidad de formas de evangelizar ese mundo.

Dada la extensión del artículo vamos a reducir la diversidad de situaciones que se reflejan en el NT a tres que, en cierto sentido pueden expresar diferentes tipos de comprensión de la relación Iglesia-mundo en el NT. En primer lugar nos referiremos a las comunidades que están insertas dentro del mundo judio; tomaremos como representante de este tipo de comunidades a la iglesia que está en el trasfondo del escrito mateano. Un segundo tipo está representado por las comunidades que se insertan en el mundo greco-latino en clara apertura a las gentes de origen pagano. En tercer lugar tomaremos una tradición que acaba desembocando en una clara ruptura con el mundo, como es la tradición joánica. Ciertamente en cada una de estas tradiciones habria que realizar bastantes diferenciaciones y matizaciones; de hecho cada una de ellas pediría una estudio monográfico. Pero no es este nuestro objetivo, puesto que lo que buscamos es constatar en los textos neotestamentarios cómo se reflejan diversas valoraciones del "mundo", asi como el pluralismo de estrategias a la hora de entrar en relación con el entorno social en el que vive la comunidad creyente. En la descripción, primero nos referiremos al contexto sociohistórico de la comunidad para posteriormente presentar la concepción del mundo y las consecuencias que eso tiene para la comunidad creyente respectiva.

\section{Comunidades en el contexto socio-religioso judio.}

Tenemos por lo menos dos escritos que están realizados en este contexto: el evangelio según S. Mateo y la carta de Santiago. Se trata de comunidades compuestas por seguidores de Jesús que son, mayoritariamente de origen judío. En gran parte se sienten integrados y continuadores de la religión judia; practican las obras de piedad propias de esta religión como son la oración (Mt 6, 5-6), la limosna (Mt 6, 1-4), el ayuno (Mt 6, 16-17); son estrictos observantes de la Ley, la cual está toda vigente, en sus más minimos detalles y hasta el final del mundo (Mt 6, 17-19); en la medida de lo posible tratan de integrase en el medio socio-religioso sin provocar situaciones críticas estériles, pero al mismo tiempo con una clara conciencia de la novedad y de la libertad que procede del acontecimiento Cristo (Mt 17, 24-27).

Sin embargo el mundo judío en Palestina sufre un terrible quiebre a partir de la guerra de finales de la década del sesenta y la destrucción de Jerusalén en el año 70; deja de existir el Templo como lugar central de la fe judia y con ello desaparece la centralidad religiosa y política que habian tenido diversos grupos e instituciones 
hasta ese momento (sacudeos, sumos sacerdotes, sanedrín); emerge la hegemonía de los fariseos y aparece como problema crítico la autoridad y la definición de la identidad de la religión judia; se endurece la posición frente a los "grupos heréticos" dentro del judaísmo; se reglamente el culto; se trata de clarificar del canon de las escrituras, asi como la interpretación de la Biblia...; es decir, se desarrolla todo aquello que va a permitir a la religión judía vivir sin la institución central del Templo; esto coloca en una situación muy crítica a las comunidades cristianas que se sienten en continuidad con el judaísmo al mismo tiempo que proclaman la novedad de Jesús como el Cristo; finalmente son marginados de la comunidad judia y tachados como herejes peligrosos.

La comunidad mateana busca integrarse pacíficamente, sin perder su propia identidad; este es el contexto donde se ubica el trasfondo del primer evangelio. La postura que se privilegia no es de rechazo o conflicto ( $s i$ exceptuamos la posición frente a los fariseos) sino el esfuerzo de mantener lo bueno de lo antiguo sin perder la identidad que proviene de lo nuevo; "Así todo escriba que se ha hecho discípulo del Reino de los Cielos es semejante al dueño de una casa que saca de sus arcas lo nuevo y lo viejo" (Mt 13, 52).

Cristo es presentado en continuidad con el A.T. como el nuevo Moisés que viene a ser el iniciador de una nueva alianza; esta nueva alianza también se realiza en el monte (en la presencia de Dios) y tiene rasgos muy novedosos respecto a la antigua alianza; los discípulos, junto con el pueblo suben acompañando a Jesús a la montaña; en ella Jesús toma asiento, puesto que el lugar de la presencia de Dios es su lugar natural; con esta imagen de cercanía al mismo tiempo que solemne, Jesús proclama el contenido de la nueva ley que caracteriza la alianza que él trae; no se trata de un conjunto de prohibiciones y leyes que se deben cumplir estrictamente; se trata, por el contrario de una invitación a la plenitud; la nueva ley es una invitación a la felicidad que pasa por vivir un conjunto de valores inversos a los que habitualmente se suelen vivir; el centro de la felicidad está en un espíritu pobre que es capaz de compartir todo lo que se tiene por amor, con corazón puro y misericordioso, constructor de paz auténtica; esto pasa por vivir e impulsar unas nuevas relaciones basadas en el compartir, en el servir donde los adoloridos y postergados de la historia van a ser los más beneficiados. La ley antigua no pierde sentido, pero llega a su plenitud con esta nueva ley que transforma los corazones y las relaciones; la actitud ante la ley no se reduce a un mero cumplimiento. No se trata sólo de no matar, sino de respetar al hermano en toda su dignidad e integridad sin insultarle ni pasarle a llevar en función de otros intereses; el que vive enemistado con su hermano es digno de la gehenna eterna y no puede asistir a la liturgia. Una radicalización análoga acontece con los otros mandatos de la ley como el aduiterio, el divorcio, la venganza, el comportamiento frente al enemigo. Si la ley antigua buscaba agradar a Dios y poder sobrevivir como pueblo, la nueva ley es mucho más ambiciosa; lleva implícito el deseo de impulsar al hombre hasta su plenitud según el proyecto de Dios; por ello la conclusión de esta radicalización de la ley es que "ustedes sean perfectos como perfecto es su Padre celestial" (Mt 5, 48) ${ }^{4}$.

4

Es muy sugerente que el paralelo de Lucas traduce perfecto como misericordioso o compasivo (Lc 6, 36) que es precisamente uno de los atributos que Dios recibe con más frecuencia en el A.T.; especialmente significativo es el texto de la manifestación de Dios en Ex 34, 6 
El cumplimiento de la nueva ley pasa por poner a Jesús en el centro de la vida de tal manera que no sea antepuesto por nada (Mt 10, 37-39). Esta actitud ante la vida y ante la ley va a llevar a un conflicto, de tal manera que serán perseguidos dentro de la sociedad (Mt 5, 11-12), viviendo la dificultad entre las personas más cercanas (Mt 10, 34-36).

El envío misionero refleja en buena medida la relación que la comunidad establece con su medio; como sabemos este discurso misionero aparece en los tres sinópticos; el texto mateano realiza una singular agrupación; une el envio de los doce discípulos (Mt 10, 1-5a), con las instrucciones para la predicación (Mt 10, 5b16), probablemente ajustadas históricamente al tiempo de Jesús ${ }^{5}$, seguido en tercer lugar por una serie de consecuencias que va a tener la predicación del Reino y que probablemente se ubican en la experiencia de la comunidad pospascual (Mt 10, 17-42). Comentamos algunos elementos que aparecen en este texto y que son significativos en la medida que reflejan la comprensión que la comunidad mateana tiene del mundo que le rodea y cómo se plantea la relación con él.

El encuadre de la misión viene dado por la perícopa Mt 9, 35-37; entre otras cosas refleja una mirada compasiva del entorno, de la gente y la necesidad imperiosa de hacer algo; esta urgencia se resuelve en una oración de petición al Padre para que envíe misioneros a la mies. Es notable que lo que lleva al envío misionero no es una interpretación apocalíptica de la realidad o una valoración negativa, sino una mirada compasiva ${ }^{6}$, que coloca a los enviados en una actitud de servicio y solidaridad.

Llama la atención que los destinatarios son 'las ovejas perdidas de la casa de Israel'; con mucha probabilidad refleja una situación histórica de la comunidad prepascual que sigue la práctica de Jesús de dirigirse solo a los judios enseñando en las sinagogas (Mt 10,35). Pero lo significativo en este caso es que los demás evangelistas no nos han dejado constancia de este hecho, lo cual significa que de alguna manera sigue siendo un dato relevante para la comunidad mateana; es decir, en el grupo de cristianos que están detrás de este escrito, hay una preocupación especial por la comunidad judía, con la que sienten unidos por una especial responsabilidad.

Si es lícito ver en el contenido y en la forma del envío misionero la manera como la comunidad mateana percibe el mundo que le rodea y el estilo de relación que debe mantener con él, entonces podriamos decir que ésta relación está marcada por dos grandes características: la compasión que se traduce en servicio y la firmeza de valores que se traduce en conflicto.

El contenido de la misión está centrado en el anuncio del Reino de los Cielos; los discípulos están llamados a realizar este anuncio no solo de palabra proclamando su cercanía, sino haciendo signos de liberación que expresan de forma evidente la realización del acontecimiento del Reino (cfr Mt 10, 8a). El enviado a este anuncio no debe recibir ningún salario especial puesto que ha sido un regalo que él mismo

5 Cfr R. AGUIRRE, - A. RODRIGUEZ, Evangelios sinópticos y Hechos de los Apóstoles, Estella, Verbo Divino 1992, pg. 201.

Es conveniente recordar aquí lo dicho en nota anterior sobre la compasión. 
ha recibido; esta misma es la experiencia y el apremio que vive Pablo par quien el anunciar el evangelio no es motivo de recompensa sino necesidad imperiosa (cfr 1 Cor 9,15-18). Es más, la misma evangelización se debe realizar con pobreza de medios ('sin oro ni plata, ni calderilla en las alforjas'), sin mucha provisión (ni alforja ni túnica), sin ejercicio de poder (renuncia al bastón). Todo ello coloca al evangelizador en una situación de confianza ante la providencia (cfr Mt 10, 29-31) y a merced de los destinatarios, los cuales son los que deciden si acogen, dan de beber y alimentan o no al enviado en su casa. Esto es muy coherente con la actitud pacífica que han de tener los seguidores de Jesús, en vez de enfrentar el mal con el mal, respondiendo a la violencia con violencia, se les pide que neutralicen la agresividad con la acogida y la disponibilidad del que está dispuesto a compartir, acompañar, poner la otra mejilla (cfr Mt 5, 38-42). Es decir, la salida al mundo como ejercicio de compasión pasa por ponerse a disposición de la gente, de sus necesidades; se trata de un ejercicio de vaciamiento y donación, donde el evangelio no aparece rodeado de fuerza sino como oferta humilde de plenitud y liberación. De nuevo podemos encontrar un amplio paralelismo en la experiencia paulina (Cfr 2Cor 4, 1$12 ; 12,8-10)$.

Esta relación de encuentro y de servicio a las necesidades de la gente no es un acomodamiento; implica una clara identidad y jerarquia de valores propia del Reino; esta jerarquía queda lapidariamente sintetizada en el discurso del monte al cual ya hemos hecho referencia anteriormente. Esta clara identidad, aunque expresada con humildad y con talante servicial lleva a experimentar el conflicto con el mundo de tal manera que los discípulos son enviados como 'ovejas en medio de lobos' (Mt 10, 16); la presencia de los seguidores de Jesús en ese medio plantea un conflicto de intereses (en el fondo de valores) que se resuelve en rupturas, persecuciones y odios, incluso entre las personas más cercanas (cfr Mt 10, 17.21-23.34-36). El mantenimiento de esta tensión tiene una doble fidelidad: fidelidad al mundo (cfr Mt 9, 36) y fidelidad a Jesús, el cual sufrió también este mismo rechazo; de alguna manera parece que esto se convierte en signo de un auténtico seguimiento de Jesús (cfr Mt 10, 24-25). Sin esta doble fidelidad no se entiende el conflicto y la persecución. Parece que todo discípulo va a tener que vivir esta conflictividad por lo cual debe desarrollar una espiritualidad de la renuncia centrada en el amor absoluto al Señor (Mt 10, 37-38), siendo capaz de relativizar todo lo demás y asumir el conflicto y la contradicción correspondientes.

Ante el evidente rechazo que la comunidad judía hace de la 'Buena Noticia' (cfr Mt 11, 20-24) y en medio de un contexto conflictivo es donde el mundo de los destinatarios de la comunidad mateana se amplia, salta las estrechas fronteras del judaísmo y se abre al mundo de los paganos; este paso en la mirada comunitaria está muy bien reflejado en varios pasajes y personajes. Asi el relato de los 'reyes magos' (cfr Mt 2, 1-12); en la fe del centurión se expresa la capacidad de creer en la Palabra de Jesús sin ver; ante la fe de este pagano Jesús exclama: "Les aseguro que en Israel no he encontrado en nadie una fe tan grande; y les digo que vendrán muchos de oriente y occidente y se pondrán a la mesa con Abraham, Isaac y Jacob en el reino de los Cielos, mientras que los hijos del Reino serán echados a las tinieblas de fuera" (Mt 8, 10b-11a). La fe de la siro-fenicia hace saltar los prejuicios religiosos y las fronteras de la evangelización (Mt 15, 21-28). Al pie de la cruz, es el centurión el que da testimonio de la divinidad del Señor (Mt 27,54). Por ello la comunidad mateana está preparada para dirigirse a todas las gentes "bautizándolas en el nombre del 
Padre y del Hijo y del Espíritu Santo" (Mt 28, 19).

La tensión que se origina en la estructuración de la tradición mateana presenta un triple desafío. Por una parte mantener la continuidad con el Antiguo Testamento, pero mostrando que la realización plena de su sentido está en Jesús. En segundo lugar está el afrontar el problema del retraso de la parusía y la amenaza de un relajamiento en la vivencia de la radicalidad del evangelio ${ }^{7}$. En tercer lugar está la clarificación de los destinatarios de la 'Buena Noticia' y el paso de los judios a los paganos.

Se trata de una comunidad profética y misionera, organizada en torno a la comunidad local pero que cuenta con 'predicadores itinerantes' de estilo carismático, que ayudan a mantener la referencia del desprendimiento y la radicalidad del seguimiento, pero que al mismo tiempo plantean el problema del verdadero y falso profetismo, es decir, el problema de los profetas que no producen el fruto que el Señor desea aunque prediquen, expulsen demonios y hagan milagros en su nombre (cfr Mt 7, 15-23); Mateo los trata como lobos rapaces vestidos con piel de oveja, agentes de iniquidad (cfr 7, 15.23); es probable que el conflicto se suscite en torno a la interpretación del A.T. (cfr 5, 17-19) y el escándalo que eso produce en los pequeños (cfr 18,6). En cualquier caso esto pide tener una autoridad dentro de la comunidad que permita zanjar estas tensiones. Asi junto a los profetas, en la comunidad mateana hay también escribas cristianos encargados de interpretar la Sagrada Escritura, tanto la Ley como la instrucción de Jesús (cfr 13,51) ${ }^{8}$. La comunidad también reconoce la autoridad de Pedro en la edificación de la Iglesia (cfr 16, 17). Pero la autoridad proviene de Jesús y de la identificación con él y con su misión (cfr $20,20-23$ ) y el ejercicio de la misma pasa por asumir la actitud del pequeño (cr. 18, 1-4) y del servidor (cfr 20, 27-28). Esta articulación de comunidad local con miembros itinerantes, misionera y abierta al entorno, articulada en torno a una autoridad reconocida le permite mantener una síntesis históricamente exitosa entre los diferentes polos de las tensiones anteriormente descritas. Se trata de una sintesis integradora que al interior de la comunidad supone buscar y servir al pequeño, al perdido, con un ejercicio constante de la misericordia entre los hermanos como símbolo de la misericordia que Dios ha tenido con todos (cfr 18, 12-18.21-35); hay conciencia que la comunidad misma es el sacramento de la presencia del Señor (cfr 18,$19 ; 28,20 b$ ). Hacia el exterior, la comunidad se compromete en una actividad misionera basada en el ejercicio de la compasión que impulsa a llevar el evangelio a todas las naciones.

\section{Comunidades en el contexto del mundo greco-latino.}

Nos vamos a referir en este apartado a un conjunto de comunidades que, aunque teniendo características bien diferenciadas comparten algunos rasgos comunes muy importantes para el tema que estamos desarrollando y que nos permite introducirlas en una tipología característica. Se trata de comunidades insertas en el mundo pagano, alejadas de la órbita de influencia del judaísmo y que reclaman la 
libertad frente a la reglamentación legalista del A.T.; parece que en su mayoría están compuestas por personas venidas del paganismo o de judíos con fuerte influencia helenista; son comunidades que buscan insertarse en el mundo greco-latino como el ámbito propio de la vida de la comunidad creyente. De una u otra manera, todas las tradiciones que en el NT están reflejadas en este tipo, se encuentran bajo la influencia de Pablo. Por una parte tenemos todas las comunidades fundadas o visitadas por Pablo y con las que el apóstol mantiene comunicación epistolar (recordemos que se trata de 14 de los 27 escritos del NT); pero también podemos integrar en el mismo tipo la comunidad que está en el trasfondo del escrito marcano y los destinatarios de la obra de Lucas. Efectivamente, tanto Marcos como Lucas han sido colaboradores directos de Pablo y han participado en su actividad misionera ${ }^{9}$; en ambos casos se trata de comunidades que viven en contextos sociales y religiosos alejados del judaísmo y abiertos a las influencias de la cultura greco-latina.

El mundo greco-latino en el que están insertas estas comunidades se caracteriza por ser una sociedad pujante, bien organizada bajo la tutela del Imperio Romano, con buena intercomunicación. Tiene una fuerte tradición en el desarrollo del pensamiento como se refleja en la obras clásicas de los grandes filósofos de la época. Pero no es un mundo unificado. Conviven diversidad de filosofías y comprensiones del mundo, diversidad de culturas, múltiples religiones junto con la religión oficial del Imperio Romano. La sociedad está fuertemente estratificada, tanto en la dignidad civil de los habitantes ante la ley, como en las diferencias socioeconómicas. Esta pluralidad y fragmentación se refleja también en la conducta moral de la población.

En este contexto, las comunidades cristianas que están dentro de esta tipología que estamos desarrollando se caracterizan por una activa inserción social, participando de las relaciones propias de esa sociedad al mismo tiempo que mantienen su identidad creyente y buscan dar testimonio de su fe manteniendo una actitud misionera. Esta actitud misionera se realiza tanto a través del ejemplo que dan las comunidades, como a partir de la predicación. Parece que la estrategia de expansión se realizó a partir de la estructura familiar.

El camino que realizó este tipo de comunidades es una de las claves que nos permite comprender cómo el cristianismo, que inicialmente fue un movimiento carismático, intrajudío y con un fuerte grado de contestación social y radicalización ética, saltó esas fronteras socio-religiosas iniciales y se instaló en el corazón de la

g

La relación entre Marcos y Pablo parece bastante cercana en los textos del N.T., aunque con algunos altibajos y tensiones. Marcos es primo de Bernabé (Col 4,10); Pablo y Bernabé le eligen a Marcos para que les acompañe (Hch 12, 25); ambos participan en el primer viaje misionero de Pablo aunque son abandonados por Marcos (Hch 13, 13), por lo que Pablo no lo elige para su segundo viaje misionero, lo cual conlleva una tensión entre los misioneros (Hch 15, $37 \mathrm{ss}$ ); sin embargo, pasados los años nos encontramos a Marcos junto a Pablo en Roma (Col 4, 10; Flm 24); incluso Pablo pide expresamente que le hagan llegar a Marcos puesto que es un buen evangelizador $(2 \operatorname{Tm} 4,11)$.

Lucas también aparece en los textos del NT como colaborador directo de Pablo. Tal vez la huella más extensa se refleje en los llamados 'fragmentos nosotros' del Libro de Hechos de los Apóstoles $(16,10-17 ; 20,5-15 ; 21,1-8 ; 27,1-28,16)$. Aparece acompañando a Pablo en su reclusión de Roma (Col 4, 10.14; FIm 24); en la segunda cautividad de Pablo Lucas es el único que lo acompaก็ล $(2 \operatorname{Tm} 4,11 ; \operatorname{Hch} 27,1 \mathrm{~s})$. 
civilización más importante del momento, consiguiendo un cierto grado de acomodamiento y aceptación en el nuevo marco social.

Partamos de un texto significativo de la tradición lucana que se enmarca dentro del proceso que hicieron estas comunidades en su acercamiento a la sociedad pagana; se trata del conocido pasaje de la conversión y bautismo del centurión Cornelio y su familia según está narrado en el capítulo diez de $\mathrm{Hechos}^{10}$. En la primera presentación del personaje Cornelio (v. 1-2) aparecen sus rasgos esenciales para la comprensión de la narración:

a) Se trata de un hombre público que cumple una función social al servicio de una estructura política y militar externa al pueblo judio; vive en una ciudad fundada en honor del Cesar y lugar de residencia de la autoridad romana.

b) Tiene una gran sensibilidad religiosa que se traduce en obras de piedad, cercano a las necesidades de la gente y de fuerte espiritualidad ('oraba constantemente a Dios'). Sin embargo se trata de un hombre impuro según la tradición judía.

c) Vive en un contexto familiar; su familia comparte con Cornelio la sensibilidad religiosa. A tenor de la narración posterior, parece que Cornelio es el 'paterfamilias' en torno a quien se organiza la casa.

A continuación nos encontramos a Pedro, quien venciendo inicialmente sus resistencias (todavia tímidamente), se pone en camino para visitar la casa de un pagano (v. 28); Cornelio aparece en su residencia, junto con sus parientes y amigos íntimos esperando la llegada del ilustre visitante y de su séquito. En el contexto familiar de la casa pagana, acontece el encuentro, la instrucción, la efusión del Espíritu Santo y la celebración del bautismo. Nos imaginamos que posteriormente a este acontecimiento, la casa de Cornelio se convertiría en un lugar habitual de reunión de las personas que en ella se habían bautizado; así mismo es probable que se seguiría utilizando para acoger a los predicadores venidos de otros lugares; si esto fue asi, la casa de Cornelio pasaria a ser un centro misionero de irradiación de la 'Buena Noticia'.

No estamos ante un caso aislado en el NT. La estructura social de la casa aparece con frecuencia y con un claro significado en relación con la organización y la tarea de la Iglesia. Son los textos neotestamentarios que están relacionados con el grupo de comunidades a que hacemos referencia en este apartado, los que traen una información más abundante relacionando la casa doméstica con la Iglesia. Vamos a repasar brevemente la información que nos ofrecen estos textos ${ }^{11}$. Con frecuencia aparece la fórmula "NN y (toda) su familia", tal y como hemos visto en el caso de la narración de Cornelio. En la primera llegada al continente Europeo, Pablo y sus acompañantes se encuentran con Lidia, rica comerciante de Filipo, quien los recibe en su casa; "ella y los de su casa recibieron el bautismo" (Hch 16, 15);

La intención redaccional del texto parece que apunta más bien a la conversión de Pedro dando cabida dentro del nuevo movimiento religioso a los que estaban excluidos por motivos legalistas. Para un estudio en profundidad del significado de esta narración se puede consultar J. RIUSCAMPS, De Jerusalén a Antioquia. Génesis de la Iglesia cristiana. Comentario lingüistico y exegético a Hch 1-12, Córdoba, El Almendro 1989, pgs 258-271. 
posteriormente parece que la casa de Lidia se convierte en lugar de encuentro y oración de la comunidad creyente (Hch 16, 40). En la misma ciudad acontece la conversión del carcelero a quien se le anuncia la Palabra del Señor junto a todos los de su casa; "inmediatamente recibió el bautismo él y todos los suyos" (Hch 16, 33). Muy significativo es el texto de 1 Cor 16, 19b: "Les envian muchos saludos Áquila y Prisca en el Señor, junto con la Iglesia que se reúne en su casa". Es decir, la casa de este matrimonio misionero es el lugar del encuentro de la comunidad creyente. Esta misma construcción lingüística aparece en el saludo que Pablo dirige a Nifas y "a la Iglesia de su casa" $(\mathrm{Col} 4,15)^{12}$. En este contexto en que se pone en relación casa, evangelización e Iglesia, aparece también el nombre de la familia de Estéfanas, que está al servicio de los santos y a quien los miembros de la comunidad deben mostrarse sumisos (1Cor 16,15-16); es Pablo mismo el que ha bautizado a esta familia (1Cor 1,16$)$. En la carta a Filemón se menciona también la Iglesia que se reúne en su casa (FIm 2).

El capítulo 16 de la carta a los Romanos resulta una fuente de información especialmente importante para nuestro tema ${ }^{13}$. Notemos, en primer lugar, la existencia de familias completas convertidas al cristianismo y en cuyas casas se reúne la comunidad creyente; tal es el caso, ya presentado anteriormente, de Áquila y Prisca (v 5), la familia de Asíncrito y Flegonta ( $v 15$ ) y la familia de Filólogo y Julia (v. 16). Pero no siempre saluda a la familia completa; hay una fórmula que se repite: "saluden a los de la familia NN"; así por ejemplo en los versículos 10 y 11 se dice "saluden a los de la casa de Aristóbulo(...) a los de la casa de Narciso"; la misma fórmula se repite en Flp 4, 22: "Os saludan todos los santos, especialmente los de la casa de César"; en estos casos el saludo se dirige hacia miembros de la familia distintos de la cabeza, que ocupan rangos inferiores en la jerarquizada estructura familiar. Esto tiene dos connotaciones importantes; por una parte expresa que la conversión no era necesariamente de toda la familia; en ocasiones sucedia que era una parte la que se convertia; el saludo iba entonces a los miembros convertidos del grupo; sin embargo, Pablo sigue manteniendo la referencia a toda la familia, es decir, la unidad familiar se conserva como realidad socialmente significativa e importante; el convertirse en cristiano no significaba necesariamente romper con los lazos de una familia no cristiana ${ }^{14}$. En otros términos, este dato nos pone en la pista que la estrategia evangelizadora realizada en la sociedad greco-latina es respetuosa de sus estructuras sociales básicas, de tal manera que la conversión no rompe con esas relaciones sociales.

Parece que esta forma doméstica de la Iglesia evolucionó rápidamente puesto que en las cartas pastorales desaparece la fórmula "Iglesia que se reúne en la casa de NN" y aparece la expresión "casa de Dios que es la Iglesia de Dios vivo, columna

Aunque no está en relación directa con nuestro tema, no deja de llamar la atención el papel importante que cumplen las mujeres en la organización y construcción de la lglesia en este período inicial.

Se duda sobre la autoria de este capitulo, pero el resultado de esta disputa no afecta mayormente a las consecuencias que tiene para nuestro tema, puesto que en cualquier supuesto resulta valiosa la información que nos brinda. propósito del matrimonio formado por un cónyuge cristiano y otro no (cfr 1 Cor 7, 12-16) 
y fundamento de la verdad". Se habla de la casa del obispo y de los diáconos (1Tm $3,4.12)$, hay referencias a otras casas $(2 \mathrm{Tm} 1,16 ; 4,19)$, pero en estos casos ya no se menciona la casa junto a la Iglesia, es decir, se refleja ya una separación entre la casa de la persona notable y la comunidad cristiana.

¿Cuál fue el motivo por el que estos primeros misioneros cristianos se orientaran hacia la casa como estrategia de evangelización? La respuesta apunta en dos direcciones. Por una parte el rechazo del judaismo expresado en la no acogida en la sinagoga impulsó a buscar otras alternativas de encuentro y de proclamación de la Palabra del Señor. Por otra parte la casa es un centro neurálgico en la estructura social de la cultura greco-latina. Efectivamente, esta sociedad se estructura a partir de dos núcleos centrales: el estado y la casa. El mismo estado es considerado como una extensión de la casa; en este sentido dice Filón de Alejandría: "si una casa es una ciudad en pequeño y si la economía (administración de la casa) se emparienta con la política, se puede decir que una ciudad es una casa grande y la política la economía de una comunidad"15. Por casa no sólo se entiende el edificio o lugar físico en el que se habita, sino la familia en su conjunto y las relaciones que dentro de ella se entablan ${ }^{16}$; se trata de una familia de tipo jerárquico-patriarcal presidida por el 'paterfamilias'u 'oikodespotes'; se compone, además, de la esposa, de los hijos, de otros familiares, del servicio y de los esclavos; se trata de un ámbito educativo, productivo y de participación social que permite la integración dentro de la sociedad; la familia es la escuela en la que se aprende a asumir responsabilidades, a gobernar, a cumplir la función social que le corresponde a cada uno. Por ello, una garantía para un servicio público eficaz es que la persona sea capaz de cumplir bien con su rol dentro de la familia; en este sentido se puede comprender el texto $1 \mathrm{Tm} 3,1-7$. Por tanto con la elección de la casa como lugar neurálgico para el primer desarrollo de la Iglesia en esta sociedad se está buscando incidir en una de las estructuras sociales básicas de mayor significado, proyección e influencia dentro de esa cultura.

Concluyendo este apartado de la relación que se da entre Iglesia y mundo dentro de las comunidades que se ubican en lo que hemos llamado órbita paulina podemos decir:

a) Al asumir la estructura social de la casa como elemento importante en el desarrollo de la primera Iglesia se está realizando una estrategia diferente

Mencionado en R. AGUIRRE, Del movimiento de Jesús ..., o.c., pg. 101.

En el libro de "La Política" de Aristóteles nos encontramos con la siguiente descripción de casa y sus relaciones en el marco de la cultura griega:

"Una vez que hemos puesto de manifiesto de qué partes consta la ciudad, tenemos que hablar, en primer lugar, de la administración doméstica, ya que toda la ciudad se compone de casas. Las partes de la administración doméstica corresponden a aquellas de que consta a su vez la casa, y la casa perfecta consta de esclavos y libres. Ahora bien, como todo se debe examinar por lo pronto en sus menos elementos, y las partes primeras y mínimas de la casa son el esclavo y el amo, el marido y la mujer, el padre y los hijos, habrá que considerar respecto de estas tres relaciones qué es y cómo debe ser cada una, a saber: la servil, la conyugal y la procreadora" (mencionado en R. AGUIRRE, Del movimiento de Jesús..., o.c., pg 97) 
a la que se tenia en la práctica de las comunidades palestinenses; estamos lejos de aplicar la ruptura familiar que late en varios textos sinópticos (Mc 3, 31-35; 10, 29-30; Mt 8, 21-22; 10, 34-37), donde el seguimiento de Jesús parece que pasa por el alejamiento de la familia y la constitución de una nueva familia. Se da por tanto un paso creativo y adaptativo a la nueva situación social.

b) Hay una valoración positiva de la nueva sociedad en la que se están insertando, puesto que tratan de respetar sus estructuras básicas y canalizan la actividad misionera a través de esas estructuras sociales. Hay también un deseo que el evangelio sea socialmente significativo a través de las estructuras naturales de esa sociedad.

c) Probablemente la casa como lugar de reunión y misión de la comunidad cristiana supuso un paso muy importante a la hora de diferenciar al cristianismo del judaísmo.

d) Al asumir la estructura de la casa, el movimiento cristiano impulsado por estas comunidades asumió también la estructuración social que se daba en la cultura greco-latina; asi las diferencias de dignidad social, el diferente acceso a la participación social, la forma de producción basada en la esclavitud, la estructura de autoridad patriarcal...

e) Sin embargo no se renuncia a los principios del evangelio, de tal manera que en Cristo ya no hay hombre ni mujer, señor o esclavo, sino que todos somos igualmente hijos de Dios y herederos del Reino (Gal 3, 27-28; Col $3,10-11)$. En este sentido es muy importante la exhortación paulina de no conformarse con los valores de este mundo, sino de mantener siempre la mente renovada para distinguir la voluntad de Dios $(\mathrm{Rm} 12,2)$.

\section{Las comunidades juánicas ${ }^{17}$.}

Los escritos juánicos ponen de manifiesto que estamos ante una tradición redaccional del NT bien caracterizada y diferenciada del resto. El texto es la expresión de la lectura creyente (es decir, a la luz del Resucitado e impulsados por su Espíritu) que hicieron esas comunidades de las experiencias y acontecimientos que les tocaron vivir. Como era de esperar, esta originalidad en la experiencia y en la redacción ponen de manifiesto una peculiar forma de comprender el mundo que les rodea, de la inserción como comunidad creyente dentro de ese mundo y de su misión. Conviene que primeramente presentemos de forma breve la experiencias centrales que marcaron la vida de estas comunidades para después pasar a describir de forma sintética las características de la tradición juánica y la relación que entabla con el entorno.

Siguiendo a Brown ${ }^{18}$ podemos delinear la historia de la comunidad juánica en

El vocabulario es un tanto impreciso a la hora de nombrar el grupo de creyentes que está en el trasfondo de los escritos juánicos; tal vez, y propiamente, hay que hablar de una tradición juánica que se va realizando en diversas comunidades, en pluralidad de lugares, evolucionando en el tiempo y recibiendo diversas influencias. juánica, Salamanca, Sígueme 1987. 
torno a las diferentes rupturas que fue sufriendo a lo largo de una efervescente y rápida evolución. Inicialmente la comunidad está formada por grupos provenientes de diferentes tradiciones palestinenses, unidas en torno a Jesús resucitado pero con características distintas, sobretodo en su relación con el judaísmo y con el Templo. Esta primera confrontación y la necesidad de una síntesis dio como resultado la formulación de una cristología muy elaborada con una clara manifestación del Cristo exaltado y preexistente y una comprensión de la comunidad que, coherentemente consistente con esa cristología, remarca la presencia y la relación personal con el Señor a través del Espíritu; comprenden y expresan la escatología como acontecimiento presente; la base de la relación comunitaria está en la adhesión personal de cada miembro al Señor, lo que da como resultado una comunidad enraizada en una relación de amor y donde se radicaliza la igualdad entre todos sus miembros. Esta evolución que se hizo probablemente con rapidez y en un ambiente dominado religiosa y culturalmente por los judíos, produjo un fuerte conflicto con el medio. La ruptura se hace evidente y se refleja en el uso que los textos juánicos hacen del término "judío", el cual no sirve para designar una realidad étnica o religiosa, sino la maldad que se opone al proyecto de Dios ${ }^{19}$; esta ruptura probablemente lleva a una profundización y radicalización de las posturas y reflexiones que tiene la tradición juánica al mismo tiempo que al abandono físico y religioso del mundo judío. La emigración les lleva a tierras paganas, de clara influencia griega; fruto de la presencia de la comunidad en este nuevo ámbito social y cultural, surge un nuevo grupo de integrante en la comunidad, esta vez venido del mundo griego. La dificultad de la adaptación social y el probable rechazo ambiental en el nuevo contexto lleva al convencimiento de la maldad de un mundo que vive de espaldas al proyecto de Dios; esta oposición se expresa en un lenguaje dualista remarcando el polo de la luz y el de las tinieblas. El estilo, la experiencia y este contexto de la tradición juánica abre la puertas a la interpretación gnóstica y dualista de la realidad circundante y de la fe; al aislamiento con el entorno se suma la división interna que terminará en la división y en la disputa por la interpretación de la tradición juánica. Dentro de este panorama se termina de escribir el cuarto evangelio y se redactan las cartas como claves interpretativas del texto juánico. En medio de esta situación de ruptura interna agravada posteriormente por la persecución que el Imperio Romano desata contra los cristianos se escribe el libro del Apocalipsis como profecía de esperanza e invitación a la confianza y a la permanencia.

Para el tema que estamos desarrollando y con el fin de percibir la relación que la tradición juánica establece con su entorno podemos estudiar el uso del término "mundo" en esta tradición. Se trata de un término ampliamente repetido y muy significativo en Juan; de las 188 veces que aparece en el NT, 104 corresponden a los escritos juánicos y 46 a los paulinos ${ }^{20}$. En pocas ocasiones Juan se refiere con este concepto a una descripción neutral del mundo físico (Jn 11, 9; 17, 5.24; 21,25$)$ o la humanidad que habita en él (ej.: Jn 1, 9.10, etc ); la mayoría de las veces tiene una

Cfr J. MATEOS, - J. BARRETO, Vocabulario teológico del evangelio de Juan, Madrid, Cristiandad 1980 , pgs. 154-165. 1061. 
acepción teológica que implica una valoración, bien de una humanidad necesitada de ser salvada (Jn 1, 29; 3, 17, etc.) o sobretodo de un orden social contrario al plan de Dios; es precisamente esta última acepción la más extendida en los escritos juánicos. El mundo ha salido de las manos de Dios pero no es capaz de reconocerlo (Jn 1, 10), de tal manera que los hombres prefieren más las tinieblas que la luz porque sus obras son malas ( $\mathrm{Jn} 3,19)$; se trata de un orden injusto, pervertido, contrario al proyecto de Dios y que en consecuencia, odia a su Hijo (Jn 7,7) y desea aniquilarlo (Jn 12, 31) y por ello persigue a Jesús y a sus discípulos (Jn 15, 18-25; $16,2)$. Este orden perverso está dirigido por "el Principe de este mundo" (Jn 14, 30) que representa la organización socio-política asentada en la sociedad y que hace que el mal permanezca ${ }^{21}$; en este sentido, el mal en el mundo tiene un aspecto dinámico y otro estático; en su aspecto dinámico se pone de relieve la acción directa que realizan los jefes de este mundo en oposición al plan salvífico de Dios (Ap 12, 9); consecuencia de la acción eficaz de los dirigentes tenemos que el mundo de los hombres se encuentra pasivamente sometido a las fuerzas del mal. Este estado de cosas genera miedo en los creyentes en Jesús de tal manera que no se atreven a hablar abiertamente de él $(\mathrm{Jn} 7,13 ; 9,22)$ y es una amenaza para sus discipulos $(19,38 ; 20,19)$. Frente a esta situación se distingue el orden traído por Jesús que no corresponde a "lo de aqui abajo" sino a "lo de arriba" (Jn 8, 23); en consecuencia, la comunidad de los creyentes en Jesús, testigo de la verdad, está enfrentada al mundo y es odiada por él (1Jn 3, 1.13); lógicamente vive separada del mundo (Jn $15,18-20 ; 16,2 ; 17,6.14 .16)$. Por ello se pide a los hermanos jóvenes de la comunidad juánica que no amen al mundo, ni lo que hay en el mundo pues "si alguien ama al mundo, el amor del Padre no está en él" (1Jn 2, 16).

Sin absolutizar las conclusiones, podemos afirmar que en la tradición joánica hay una resistencia a mirar el entorno de forma compasiva; hay más bien una relación de enfrentamiento y una tendencia a la ruptura frente a lo diverso. Como muy bien intuye Brown es muy llamativo que una experiencia comunitaria que absolutizó la radicalidad del amor mutuo como característica esencial y central de la voluntad de Jesús (Jn 15, 12), expresión de un auténtico conocimiento de Dios (1Jn 4, 1213), signo de su elección ante los hombres y manifestación clara de haber pasado de la muerte a la vida (1Jn 3,14), sin embargo propiciase la ruptura e incluso el odio hacia los hombres que habitan el mundo, incluidos los que un día fueron hermanos de la comunidad y se separaron de la misma; en este sentido, en Juan el amor no tiene el alcance universal, sino acotado a los que creen; esto implica una mirada y comprensión del mundo muy diferente de la que se refleja en el sermón del monte cuando se invita a amar a los enemigos y se habla de un Dios que hace llover sobre buenos y malos.

Es posible que la debilidad de la relación que la comunidad juánica mantenía con su medio externo acrecentara las diferencias al interno; en una experiencia comunitaria de tipo carismático, donde todos se sentian iluminados por el Espíritu Santo, revestidos de su autoridad, aislados del entorno, el ejercicio del discernimiento resultaba complejo, de tal manera que era muy difícil evitar la ruptura, tal y como finalmente sucedió. El pequeño grupo que luchó por una interpretación no 
gnóstica de la tradición juánica, que es precisamente el que se refleja en las cartas, acabó finalmente reconociendo la autoridad de la gran Iglesia, pero al mismo tiempo consiguió integrar el principio del amor dentro del ejercicio del pastoreo como elemento central de discernimiento, con lo cual toda la Iglesia salió enriquecida.

\section{Tensiones y síntesis.}

La configuración histórica de estos tres tipos, necesariamente se realizó a través de apuestas creativas y desafiantes, que en algún momento debieron producir tensiones al interior de las comunidades. La postura integradora de la tradición mateana supone asumir la tensión de la diversidad, incluso sabiendo que no toda diversidad es buena; en este contexto es muy significativo la parábola de la cizaña, donde el dueño del campo sabe que hay mala hierba creciendo junto al trigo, pero igual la deja hasta el tiempo de la cosecha "no sea que al arrancar la cizaña se arranque también el trigo" (Mt 13, 29). Los escritos de la tradición paulina reflejan diversas tensiones en la vida de aquellas comunidades; estas tensiones se comprenden bien en el marco de la búsqueda de los caminos que llevan a la inserción social sin dejar de ser fieles al carisma recibido en el evangelio. Por parte de la tradición juánica ya hemos apuntado que las diferencias y divisiones desembocaron en una ruptura definitiva.

Las dificultades no se ubican sólo al interior de cada una de las tradiciones; hay una tensión mayor que nace de la relación entre las diversas tradiciones dentro de la comunión eclesial; reflejos de esta tensión aparecen en el concilio de Jerusalén (cfr Hch 15) y la relación, en ocasiones conflictiva, entre Pedro, Santiago y Pablo (Gal 2, 11-14). Pero al mismo tiempo estos conflictos ponen de relieve el deseo de mantenerse unidos pese a la diversidad de experiencias y tradiciones. La esencial vinculación al Señor y la continuidad de su misión por la fuerza del Espíritu, hace que estas tradiciones se sepan y se acepten mutuamente referidas y enriquecidas.

\section{Conclusiones.}

La expansión de las primeras comunidades cristianas es un proceso dinámico que influye en el medio en que viven estas primeras iglesias, pero que también afecta y transforma la propia realidad intra-eclesial profundizando en el valor y el contenido del mensaje que trasmiten. La necesaria relación que entablan estos grupos cristianos con el mundo no tiene un molde único; hay diferencias notables, al mismo tiempo que algunos denominadores comunes. Vamos a partir comentando los aspectos comunes para después centrarnos en las diferencias. Finalmente haremos algunos alcances para nuestra realidad.

a) Los tres tipos de comunidades descritas se orientan inicialmente hacia su medio entorno; en ese ámbito viven con un talante abierto y misionero. Esta experiencia misionera va trasformando en cierta medida la conciencia y la praxis comunitaria. La comunidades insertas en el medio judio se caracterizan por integración de la tradición del A.T., al mismo tiempo que toman mayor conciencia de la novedad de Cristo y del destino universal de la 'Buena Noticia', interpretando así el privilegio de la elección como responsabilidad frente a la humanidad de ser testigos de la acción salvifica de 
Dios en el mundo e invitando a todos los pueblos a su banquete ( $\mathrm{Mt} \mathrm{28}$, 19). Las comunidades insertas en el mundo greco-latino tienden a integrar estructuras esenciales de esa sociedad como es la familia y la casa como medio de evangelización; esto supone abandonar la sinagoga y las formas clásicas judias como plataforma de predicación de la 'Buena Noticia'. En las comunidades juánicas se van integrando nuevos miembros venidos de tradiciones y culturas heterogéneas, como son los samaritanos y los griegos. En este proceso de apertura, cada una de las tradiciones tiene que realizar cambios en su concepciones iniciales, saltar prejuicios y diferenciar lo esencial (la novedad de Cristo) de las formas. En este sentido, el camino misionero de estas primeras comunidades cristianas es también un camino de conversión haciả la comprensión y la adhesión a un Cristo más auténtico.

b) El contacto con el mundo, la asunción de tener una responsabilidad misionera, lleva a reforzar la identidad cristiana de las comunidades nacientes. En diálogo con el mundo pagano y judio, lejos de diluirse, la identidad de estos grupos y la claridad de la pertenencia a la Iglesia se reafirma y se profundiza. Es decir, si la evangelización supone un clara convicción de la fe que se vive y se comparte, al mismo tiempo la identidad creyente crece con la realización de la misión.

c) El configurarse con Cristo convirtiéndose en seguidores de Jesús lleva a las primeras comunidades a un estilo de vida característico que contrasta con el medio ambiente y que es consistente con la vivencia de lo que se celebra y se proclama. En las tres tradiciones asistimos a una absolutización de Cristo y una relativización de otros valores y mediaciones. Esta absolutización se traduce en un estilo de vida marcado por el compartir, por la solidaridad y por el espíritu de servicio, tanto al interno de la vida comunitaria como en su inserción en el mundo; todas estas prácticas no hacen más que traducir y concretar el principio del amor. Este estilo de vida, sintéticamente expresado en el 'sermón del monte', contrasta con los valores mayoritarios que se viven en el entorno social en el que se insertan las comunidades cristianas; mantener este espíritu hace que este grupo sea 'sal y luz' en medio del ambiente social; es decir, la comunidad se configura como 'sociedad de contraste', de tal manera que el mensaje del evangelio no coincide con ninguna de las culturas con la que interactúan.

d) La inserción en el medio, el asumir la tarea evangelizadora, supone un cierto grado de conflicto. Tanto al exterior, como al interior de cada tradición, así como en el plano de la relación que se entabla entre las diferentes tradiciones. Sin embargo, en todas las tradiciones del NT prima la conciencia de comunión, la necesidad de confluir en lo esencial, al mismo tiempo que hay un respeto y un aprendizaje en la diversidad. El proceso de encarnación del evangelio no resulta pacífico y lineal; está marcado por tensiones, rupturas, persecuciones, malos entendidos, etc.

e) La tarea evangelizadora supone un cierto grado de estructuración eclesial, diferenciación de funciones al interior de estas comunidades que viven 
como grupo minoritario y en cierta medida alternativo dentro de la sociedad mayoritaria. Pero al interior de la relación comunitaria, siendo diferenciada y estructurada, todos los miembros tienen una misma dignidad; el cambio esencial viene dado por la conversión y la celebración del bautismo que configura a la persona con Cristo y le convierte en parte de su cuerpo dentro de la historia humana; es decir, la diferenciación esencial que se hace es entre estar dentro o fuera de la comunidad creyente, al mismo tiempo que se comprende la comunidad creyente en servicio y misión hacia los no creyentes.

f) El imperativo de la evangelización no es desarrollado con un gran despliegue de medios y de fuerza; lejos de buscar una postura de dominación, al evangelizador se le pide que vaya confiadamente, poniéndose en manos de la gente, como pobre que acoge lo que le dan para subsistir y continuar ejerciendo su tarea. La fortaleza del evangelio (tal vez podríamos añadir que también es un signo de su autenticidad) se manifiesta en la debilidad del evangelizador.

Como decíamos anteriormente, el camino recorrido por estas primeras comunidades no es un todo homogéneo y uniforme; está marcado por importantes diferencias; respecto al tema que estamos tratando subrayamos las siguientes:

a) Las tres tradiciones neotestamentarias comentadas experimentan la resistencia del mundo para acoger la 'Buena Noticia', generándose diferentes niveles de conflicto y rechazo. Sin embargo la postura que adoptan las diferentes tradiciones ante este conflicto con el mundo es claramente diferente. La tradición mateana, aún siendo muy consciente de la dificultad, apuesta por la integración; no se trata de una integración indiferenciada, sino discernida desde la novedad que supone Cristo, pero sin por ello rechazar lo bueno que hay en la tradición del medio en el que vive inserta. En cualquier caso, la adhesión a Cristo y a su mensaje le lleva a una opción de amor universal, amor gratuito, que pasa también por el amor a los enemigos; todo conlleva una mirada no ingenua del entorno, pero sí una mirada compasiva, mirada que conjuga la sencillez con la prudencia, pero que en cualquier caso no renuncia a la inserción en el medio. En la tradición paulina, la inserción en el medio lleva a adoptar estructuras sociales típicas de la sociedad greco-latina, haciendo de esas estructuras y probablemente, de su forma de vida, elementos importantes en la evangelización de la sociedad; a través de esta estrategia se busca tener una cierta incidencia en la sociedad. La tradición juánica, según lo que hemos expuesto, se caracteriza por una visión muy negativa de la realidad en la que vive inserta, del mundo; esta valoración acaba llevándola a la ruptura y al aislamiento, además de propiciar una lectura dualista de la realidad.

b) Otra diferencia de estas tradiciones se ubica en la forma como integran la tradición religiosa judia. Una vez más, el caso mateano vemos una postura conciliadora e integradora, respetando la identidad del vino nuevo que representa Cristo; pero la nueva ley no niega la antigua sino que la lleva a la plenitud. En la tradición paulina se remarca la libertad que nace del en- 
cuentro con Cristo; ya ha pasado el tiempo de la ley, del intento de justificarse por las obras; con Cristo acontece la gratuidad de la salvación; la adhesión creyente al Señor es la condición de la salvación. Juan, indirectamente, también plantea una superación del A.T. El encuentro con Cristo supone un nuevo nacimiento, que no es de la carne ni de la sangre de la tradición sino del Espíritu que da vida.

c) Tanto la tradición mateana como paulina intentan integrar las comunidades cristianas en el devenir de la historia, haciéndolas protagonistas de ella al mismo tiempo que se espera su consumación. Esto implica una valoración de la misma historia, una comprensión de su consistencia y una aceptación de la historia como ámbito en el que se va desarrollando el plan de Dios. Probablemente esta conciencia crece con el retraso de la parusía y la necesidad de enfrentarse a la supervivencia a lo largo del tiempo. Sin embargo, en la tradición juánica encontramos una escatología ya realizada; lo definitivo de la promesa ya acontece; el encuentro con el Señor y la experiencia de eternidad acontece en el hoy de la fe; ya no tiene sentido hablar de la ley antigua, como tampoco tienen mucho sentido hablar de la historia, puesto que ésta ya ha llegado a su término en Cristo; tan sólo falta la escena final del duelo entre la luz y las tinieblas; lógicamente esto dificulta la inserción histórica y la permanencia en el tiempo de la misma comunidad.

d) Una tercera diferencia estaría en el grado de estructuración de la comunidad creyente; este grado de estructuración está en buena medida relacionado con la actividad misionera que realiza la comunidad. Los polos en este punto vendrían dados por la comunidad juánica por una parte (mínimo grado de estructuración) y por las comunidades que están detrás de las cartas pastorales por otra (el mayor grado de estructuración). Probablemente este grado de estructuración tiene alguna relación con la forma de concebir la historia y el quehacer de la Iglesia en medio de esa historia.

Resulta estimulante el proyectar esta reflexión al momento actual. Esta proyección supondría un desarrollo propio que excede el marco de estas conclusiones; en esta tarea, junto con lo atractivo que pueda resultar hay que considerar aspectos metodológicos puesto que la tentación de hacer paralelismos y traslaciones indebidas es grande y se trata de un error frecuentemente cometido. Con todo apunto algunas ideas iniciales. Es evidente que la relación que la Iglesia ha ido manteniendo con el mundo ha sido cambiante a lo largo de los siglos, dependiendo de múltiples factores. De forma genérica se podría afirmar que la sociedad de cristiandad supone un intento de integrar el mundo dentro de las fronteras eclesiales; este modelo hace aguas en las tierras de misión, donde los cristianos son un grupo minoritario con muy poca capacidad de influencia social; pero el modelo también ha entrado en crisis en las sociedades tradicionalmente cristianas. Dentro del ámbito católico, el siglo XIX supone el momento de mayor lejanía y mutua incomprensión entre la Iglesia y el mundo; el desarrollo del espíritu cientifico y la progresiva secularización del occidente ha ido ubicando a gran parte de la sociedad occidental al margen de la influencia eclesiástica. El s.XX ha supuesto un lento camino de reencuentro 
cuyo punto culminante por parte de la Iglesia ha sido el Concilio Vaticano II. Este concilio expresa una actitud ante el mundo caracterizada por el respeto, por la valoración y por el sincero deseo de encuentro y diálogo.

La etapa posconciliar no ha seguido un proceso ni previsible en cuanto a las expectativas, ni lineal en el desarrollo de este encuentro; el mundo con el que dialogaba el concilio ha cambiado vertiginosamente y se ha ampliado en sus fronteras. Efectivamente, por una parte las claves culturales propias de las sociedades occidentales (interlocutor privilegiado del concilio) han cambiado muy notablemente; esto se traduce en un evidente cambio de expectativas sociales que en lo referente a la religión se está traduciendo en un alejamiento de la religiosidad formal y de pertenencia institucional, pero con un notable aumento de las experiencias religiosas, produciéndose así lo que podríamos denominar un proceso de "des-secularización de las conciencias". Por otra parte, el mundo se ha ampliado notablemente, por lo menos desde la perspectiva del interlocutor eclesial; Asia y África han entrado con fuerza en el escenario del diálogo suscitando otras temáticas y expectativas diferentes de las planteadas en las décadas del concilio; América Latina se perfila como el gran continente de la esperanza cristiana, pero también aporta nuevas visiones; en las sociedades del bienestar social surge el polo tensionante y desestabilizante del llamado cuarto mundo; todo ello marcado por un proceso de subjetivización de las conciencias.

En el modo de inserción de la Iglesia en el mundo actualmente, van apareciendo con nitidez cuatro grandes posturas: 1) la añoranza del pasado y el intento de una restauración (en la medida de lo posible); 2) el dualismo pero con un talante fuertemente misionero; 3 ) la integración dentro del mundo, pero desde una postura profética; 4) la acomodación acrítica al mundo. Este análisis se complejiza más si tenemos en cuenta la inserción en la culturas que históricamente han estado fuera de la influencia cristiana.

Este mundo contemporáneo no es sin más equivalente al que encontramos en el siglo I de nuestra era; pero al igual que en aquel momento, la Iglesia pasa a ser minoritaria, va perdiendo sus cuotas de influencia social y se va encontrando en un mundo extraño que al mismo tiempo necesita evangelizar para ser fiel a su propia esencia. Sería un error el aplicar sin más las soluciones que aplicaron las primeras comunidades cristianas en la tarea de evangelizar una sociedad desconocida y plural; pero ciertamente hay mucho que aprender de las actitudes y de las orientaciones que tuvieron, así como de las diferentes situaciones y tensiones que se fueron creando por la iniciativa y acción de estas comunidades. La experiencia de la primera Iglesia nos desafia a la creatividad, a la audacia, a la permanencia y a la tolerancia. Probablemente, de la misma manera que las primeras comunidades cristianas crecieron en la medida que se relacionaron con las fronteras sociales de la fe, la Iglesia actualmente necesita seguir profundizando en ese dinamismo que le lleva a la frontera y a los márgenes sociales, saliendo de si misma para encontrarse con 'los que están fuera'; pensemos en el cambiante mundo de los jóvenes, los grandes grupos de marginados sociales, el sub-mundo de la delincuencia...Mantener la tensión de esta salida refuerza la identidad al mismo tiempo que da conciencia de la relatividad de muchas formas de pensar y de actuar que se van integrando por el camino como la formas naturales de hacer. Este encuentro con 'la frontera' desde la fe exige una buena dosis de creatividad para adecuar el lenguaje, la acciones y la 
presencia de tal manera que resulten significativas. La pluralidad de situaciones y de sensibilidades se traducirán en un sano pluralismo de estrategias y de formas de hacer; esto puede posibilitar el necesario aprendizaje, no exento de tensiones tanto al interior como al exterior.

El dejar de realizar este camino de salida y de encuentro con el mundo, una valoración excesivamente negativa o pesimista del entorno puede llevar con facilidad a situaciones de encerramiento estériles para la misión evangelizadora y de consecuencias muy negativas para la pervivencia de la misma comunidad creyente. 